Keywords: matrix metalloproteinase 2; brain tumour; gene therapy; vessel maturation; tumour invasion; glucose transporter-1

\title{
Dual roles of tumour cells-derived matrix metalloproteinase 2 on brain tumour growth and invasion
}

\author{
Ching-Fang $\mathrm{Yu}^{1,2,3}$, Fang-Hsin Chen ${ }^{2,3,4}$, Meng-Hsuan Lu ${ }^{1}$, Ji-Hong Hong ${ }^{\star 2,3,4}$ and Chi-Shiun Chiang ${ }^{\star, 1,5}$ \\ ${ }^{1}$ Department of Biomedical Engineering and Environmental Sciences, National Tsing-Hua University, Hsinchu 30013, Taiwan; \\ ${ }^{2}$ Department of Radiation Oncology, Chang Gung Memorial Hospital at Linkou, Taoyuan 33382, Taiwan; ${ }^{3}$ Radiation Biology \\ Research Center, Institute for Radiological Research, Chang Gung University/Chang Gung Memorial Hospital, Linkou, Taoyuan \\ 33302, Taiwan; ${ }^{4}$ Department of Medical Imaging and Radiological Science, Chang Gung University, Taoyuan 33302, Taiwan and \\ ${ }^{5}$ Institute of Nuclear Engineering and Science, National Tsing-Hua University, Hsinchu 30013, Taiwan
}

Background: A previous study on a murine astrocytoma cell-line ALTS1C1 showed a highly invasive pattern similar to clinical anaplastic astrocytoma in vivo. This cell-line also expressed a high level of matrix metalloproteinase 2 (MMP2). This study aimed to verify the role of MMP2 in brain tumour progression.

Methods: ALTS1C1 and MMP2 knockdown (MMP2kd) cells were inoculated intracranially, and tumour microenvironment was assessed by immunohistochemistry staining.

Results: MMP2 expression was co-localised with CD31-positive cells at invading the tumour front and correlated with an invasive marker GLUT-1. The suppression of MMP2 expression prolonged the survival of tumour-bearing mice associated with tumours having smoother tumour margins, decreased Ki67-proliferating index, and down-regulated GLUT-1 antigen. Although the reduction of MMP2 expression did not alter the vessel density in comparison to parental ALTS1C1 tumours, vessels in MMP2kd tumours were less functional, as evidenced by the low ratio of pericyte coverage and reduction in Hoechst33342 dye perfusion.

Conclusions: This study illustrated that tumour-derived MMP2 has at least two roles in tumour malignancy; to enhance tumour invasiveness by degrading the extracellular matrix and to enhance tumour growth by promoting vessel maturation and function.

High-grade gliomas (HGG), including anaplastic oligodendroglioma, anaplastic astrocytoma, and glioblastoma multiforme (GBM), are the most aggressive brain tumours. HGG has distinctive characteristics, including immoderate proliferation, high invasiveness, anti-apoptosis, enhanced neovascularisation, and suppression of anti-tumour immunity. The survival rate for patients with HGG has remained to be low despite of the improved development of surgery, radiation therapy, and chemotherapy over the past two decades (Omuro and DeAngelis, 2013; Stupp et al, 2015; Blumenthal et al, 2017). The low curing rate for HGG is mainly due to the high invasive nature of these tumours, which prevents complete surgical resection and resistance to cytotoxic therapies (Giese et al, 2003). Secretion of proteolytic enzymes, such as serine, metallo-, and cysteine proteases, from tumour cells to degrade extracellular matrix (ECM) components and other natural barriers is the key feature for their invasiveness (Egeblad and Werb, 2002; Rao, 2003).

Matrix metalloproteinases (MMPs) are a family of secreted, zincdependent endopeptidases and are involved in tissue-remodelling processes, including wound healing, embryo implantation, tumour invasion, metastasis, and angiogenesis (Stetler-Stevenson, 1990; Ray and Stetler-Stevenson, 1994; Rosenberg, 1995). Considerable evidences implicate that the MMPs, in particular MMP2 and MMP-9, are active contributors for the progression of malignant 
glioma by increasing cancer-cell growth, migration, invasion, and angiogenesis (Coussens and Werb, 1996; Sawaya et al, 1996; Forsyth et al, 1999; Guo et al, 2005; Vehlow and Cordes, 2013).

MMP2 expression is higher in gliomas than in normal brain tissue (Lampert et al, 1998; Forsyth et al, 1999). Furthermore, the brain tumours expressing higher levels of MMP2 are frequently associated with higher degrees of invasion, metastasis, and angiogenesis. The increase in MMP2 could be found in both tumour and vasculature cells, indicating multiple roles for MMP2 in tumour progression (Sawaya et al, 1996; Forsyth et al, 1999; Vehlow and Cordes, 2013).

One of the most important challenges in determining the role of MMP2 plays in glioma progression is differentiating between its various roles at each stage of tumour progression. We have previously reported that a murine astrocytoma cell-line, ALTS1C1, displays an invasive infiltrating pattern in the tumour margin similar to malignant brain tumours (Wang et al, 2012). Invasion is a process involving a cross-talk between the invading cells and the host cells. For example, we previously demonstrated that tumour-derived stroma-derived factor-1 (SDF-1) could mobilise macrophages to promote tumour invasion in both primary (Wang et al, 2012) and radiation-induced recurrent tumours (Wang et al, 2013). In addition to SDF-1, we found that ALTS1C1 cell-lines in vitro expressed even higher levels of MMP2 mRNA than astrocytes and GL261 cells, a commonly used murine glioma cell-line (Chiang et al, 2012). We took advantage of the experimental murine tumour model to examine in detail the various roles that MMP2 plays in tumour progression and invasion. We found that MMP2 may have dual roles in the development of brain tumour malignancy-to break down ECM as the tumour expands to assist tumour cell invasion into peripheral normal parenchymal tissues, and secondly to enhance vessel function by increasing pericyte coverage on vessels and thereby promoting cancer-cell proliferation.

\section{MATERIALS AND METHODS}

Mice. Male 7-to-8-week-old C57BL/6J mice were purchased from the National Laboratory Animal Center, Taiwan. For all experiments, mice were used according to the animal experimental guidelines set by and with the approval of the Institutional Animal Care and Use Committee of National Tsing Hua University, Taiwan (IACUC:10012).

Cell line cultures. Murine ALTS1C1 (BCRC60582, Taiwan) cellline was originally established in our lab (Wang et al, 2012) and maintained in Dulbecco's modified Eagle's medium (Invitrogen) with $10 \%$ foetal bovine serum (Invitrogen), $1 \%$ penicillinstreptomycin (Invitrogen) and was incubated at $37^{\circ} \mathrm{C}$ in a humidified $5 \% \mathrm{CO}_{2} /$ air atmosphere.

Plasmids construction, virus production, and infection. Lentiviral short hairpin RNA (shRNA) expression vector (RNAi Core) was used for gene knockdown. Three small interfering RNA (siRNA) sequences were referred to the published references against MMP2 gene expression (Mosig et al, 2007; Gerg et al, 2008; Droppelmann et $a l, 2009)$. The information of siRNA sequences and vector construction was described in supplementary material and method section. Virus production and infection were according to the manufacturer's protocol. Briefly, 293TN cells were co-transfected with vector and packaging plasmids by Lipofectamin2000 (Invitrogen) and incubated overnight. The cells were replaced by a fresh medium for viral production. ALTS1C1 cells were infected with media containing $20 \%$ of a viral medium and $8 \mu \mathrm{g} \mathrm{ml}^{-1}$ polybrene (Sigma-Aldrich) for $48 \mathrm{~h}$ and selected by $8 \mu \mathrm{g} \mathrm{ml}^{-1}$ puromycin (Invitrogen) for 10-14 days. The stable transfected cells were named as ALTS1C1-MMP2kd1, ALTS1C1-MMP2kd2, and ALTS1C1MMP2kd3.
Gelatin zymography. ALTS1C1 and ALTS1C1-MMP2kd cells were incubated for $24 \mathrm{~h}$ for examination of the MMP2 activity by gelatin zymography. Culture-conditioned media were electrophoresed on a $10 \%$ SDS-polyacrylamide gel containing $1 \mathrm{mg} \mathrm{ml}^{-1}$ gelatin. After electrophoresis, the gel was rinsed, renaturated, and stained with coomassie brilliant blue R-250 for MMP2 activity quantification (Frankowski et al, 2012).

Cell invasive and migration assay. Cell-invasive assay and migration assay were performed by BD BioCoat Matrigel invasion chambers and $\mathrm{BD}$ BioCoat cell culture insert according to the manufacturer's protocol. Briefly, $2.5 \times 10^{4}$ ALTS1C1 or ALTS1C1MMP2kd cells suspended in a medium containing 2.5\% FBS were added into the upper inserts, and a medium containing 10\% FBS was added to the wells of a plate. After $18 \mathrm{~h}$, non-invading or nonmigrating cells were removed by scrubbing with a cotton swab. The cells were fixed by methanol, stained with Giemsa solution, and counted by ImageJ software.

Orthotopic intracranial tumour implantation. ALTS1C1 or ALTS1C1-MMP2kd cells $\left(1 \times 10^{5}\right.$ cells $)$ were inoculated intracranially to mice. The procedure of I.C. tumour implantation was the same as described in a previous publication (Wang et al, 2012). When the signs of a neurologic deficit (lethargy, failure to ambulate, or loss of $>20 \%$ of body weight) were shown, the mice were killed. Brain tumour samples were removed, embedded in an OCT compound (Sakura Finetek), and stored at $-80^{\circ} \mathrm{C}$.

Immunohistochemical staining (IHC). Tumour hypoxia and blood flow were assessed by injecting pimonidazole (PIMO, $160 \mathrm{mg} \mathrm{kg}^{-1}$, HPI) and Hoechst33342 (10 $\mathrm{mg} \mathrm{kg}^{-1}$, Invitrogen) into mice $1 \mathrm{~h}$ and $5 \mathrm{~min}$, respectively, before sacrifice. Frozen tissue sections were stained with antibodies against CD31, CD4, CD8, Gr-1, caspase-3 (BD Phamingen), NG2, LC3B (Millipore), VEGF, MMP2, GLUT-1, Ki67 (abcam), F4/80 (Bio-Rad), and anti-PIMO (HPI). Tissue sections were mounted with PBS containing 1\% BSA and $0.01 \%$ Tween-20 for $40 \mathrm{~min}$ to prevent non-specific binding and increase the permeability. Primary antibodies were detected using secondary antibodies labelled with streptavidin peroxidase (HRP), Alexa Fluor 488, or Alexa Fluor 594 (Invitrogen). The images were processed and analysed by Image-Pro Plus 6.0 software and ImageJ $1.48 \mathrm{v}$ software.

Statistics. The two-tailed Student's $t$-test or one-way ANOVA were used to determine the statistical significance of differences and performed using GraphPad Prism. The differences were considered to be statistically significant when $P$-values were $\leqslant 0.05$.

\section{RESULTS}

MMP2 expression is correlated with tumour invasion. Our previous study showed that ALTS1C1 tumours are highly invasive, which are associated with tumour-secreted SDF-1 (Wang et al, 2012). In addition to high SDF-1 expression levels in tumour cells, array data show that MMP2 is also highly expressed in ATLS1C1 cells (Chiang et al, 2012). MMP2 has been reported to be associated with migration and invasion of malignant gliomas. To evaluate the role of MMP2 in brain tumour progression, MMP2 expression in the tumour invasion front and in the tumour core was assessed by IHC in orthotopic ALTS1C1 tumours. We found that many MMP2 along with the invasion marker, glucose transporter-1 (GLUT-1), at invasion tumour fronts and infiltrating islands were co-localised with $\mathrm{CD} 31^{+}$vessels (Figure $1 \mathrm{~A}$ and $\mathrm{B}$, respectively). Extremely less MMP2 or GLUT1 signal was found to co-localise with $\mathrm{CD}_{31}{ }^{+}$vessels in the tumour core (Figure 1C). However, this result cannott conclude if the MMP2 detected on CD31-positive cells is secreted by the tumour cells, TAMs, or 
A
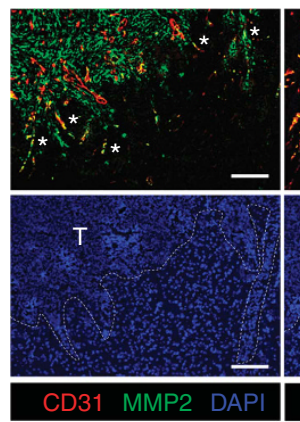

C
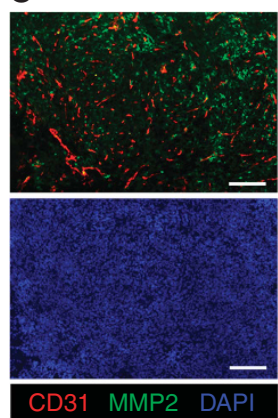

B

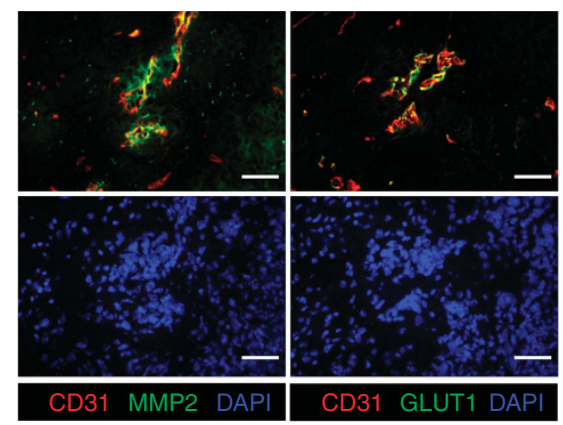

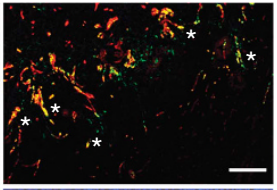
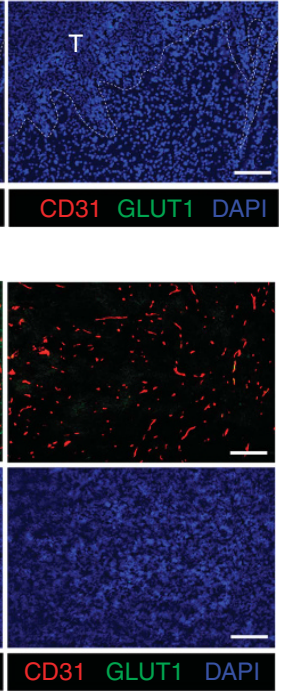

Figure 1. The association of MMP2 and GLUT-1 expression with tumour invasion in an orthotopic ALTS1C1 tumour model. The co-localisation of MMP2/CD31 and GLUT1/CD31 in ALTS1C1 tumours was stained by fluorescent IHC staining. Representative images of MMP2/CD31 and GLUT1/ CD31 staining at (A) invasion tumour fronts, $(\mathbf{B})$ infiltrating islands, and (C) the tumour core. The star indicated the co-localisation of MMP2 or GLUT1 on CD31-positive cells. Scale bar: $200 \mu \mathrm{m}$ in $\mathbf{A}$ and $\mathbf{C}$, and $50 \mu \mathrm{m}$ in B.

endothelial cells. Nevertheless, this result illustrates that brain tumours at the invading fronts have a distinct tumour microenvironment compared to those in the tumour core.

MMP2 knockdown decreases cell invasion and prolongs the survival of brain tumour-bearing mice. To examine the role of MMP2 in tumour invasion, MMP2 expression in ALTS1C1 cells was suppressed by the shRNA system. Inhibitory efficiency and specificity in transfected cell-lines was confirmed by RT-PCR, Q-PCR, and gelatin zymography (Supplementary Figure S1). The candidate sequence shRNA2 was the most effective fragment to suppress MMP2 expression. Therefore, the shRNA2-transfected cell-line (named briefly as MMP2kd) was used in the following experiments. The results show that the knockdown of MMP2 expression does not affect the in vitro cell growth kinetics (Figure $2 \mathrm{~A}$ ) or cell migration rate, as assessed by Boyden chamber assay (the left panel of Figure 2B), suggesting that cancer cellderived MMP2 does not have a direct growth or migrationstimulating effect on ALTS1C1 cancer cells. However, the Matrigel invasion assay showed a significant reduction in the invasive ability of MMP2kd cells (14.7 \pm 0.6 counts per field) compared with parental cells $(48.1 \pm 11.7$ counts per field; right panel of Figure $2 \mathrm{~B}$ ), supporting the view that cancer cell-derived MMP2 activity might be an essential factor that directly contributes to the invasiveness of ALTS1C1 astrocytoma tumours. The transfection of control shLuc had no effect on in vitro cell double time, migration, and invasion rates (Supplementary Figure S2A and B), and neither on the in vivo tumour growth rate (Supplementary Figure S2C).

To further explore the role of cancer cell-derived MMP2 on tumour growth in vivo, the survival of mice carrying ALTS1C1 or MMP2kd cells was measured in an orthotopic tumour model. The Kaplan-Meier survival curves showed that mice-bearing intracranial MMP2kd tumours survived longer than mice-bearing parental ALTS1C1 tumours (Figure 2C). The longest survival period of
MMP2kd brain tumour-bearing mice was extended to 46 days. The median survival period of 43 days for MMP2kd tumour-bearing mice was longer than 25 days observed for ALTS1C1 tumourbearing mice. MMP2 IHC staining (Figure 2D) confirmed the lower MMP2 activity in MMP2kd tumours, as compared to the parental ALTS1C1 tumours, which was further confirmed by zymography (Supplementary Figure S3).

The in vitro cell growth curve (Figure $2 \mathrm{~A}$ ) has shown that the suppression of MMP2 expression does not affect cell proliferation rate. Therefore, we were interested to know if the prolonged survival for mice-bearing MMP2kd tumour is associated with the kinetics of tumour growth in vivo, brain tumour-bearing mice were killed on specific days following tumour implantation (day 10 , day 17 , and day 24) or on the day when the mice showed signs of neurological deficit, and the maximum tumour cross sections of these tumours were analysed by H\&E stain. The data (Figure 3A) show that ALTS1C1 and MMP2kd tumours were similar in size during the early stages (day 10 and 17), but after 24 days, the maximum area of MMP2kd tumour cross sections $\left(8.8 \pm 0.3 \mathrm{~mm}^{2}\right)$ was significantly smaller than those of parental ALTS1C1 tumours $\left(15.0 \pm 0.7 \mathrm{~mm}^{2}\right)$. This indicated that MMP2kd tumours had a slower tumour growth rate compared with ALTS1C1 tumours between 17 and 24 days. These results suggested that MMP2 suppression might not have any effect on initial tumour growth, but could affect the later phase of tumour growth. However, when the tumours from endpoint mice (about 25 days from ALTS1C1 tumour-bearing mice compared to about 43 days of MMP2kd tumour-bearing mice) were compared, MMP2kd tumours were found to have significantly larger tumour sizes than ALTS1C1 tumours (last panel of Figure 3A). It appears that the slower tumour growth rate for MMP2kd tumours is not associated with cell death because these two tumours have a similar index for Caspase-3 (a marker for apoptosis) and LC3B (a marker for autophagy) (Supplementary Figure S4). This finding indicates that mice-bearing MMP2kd 

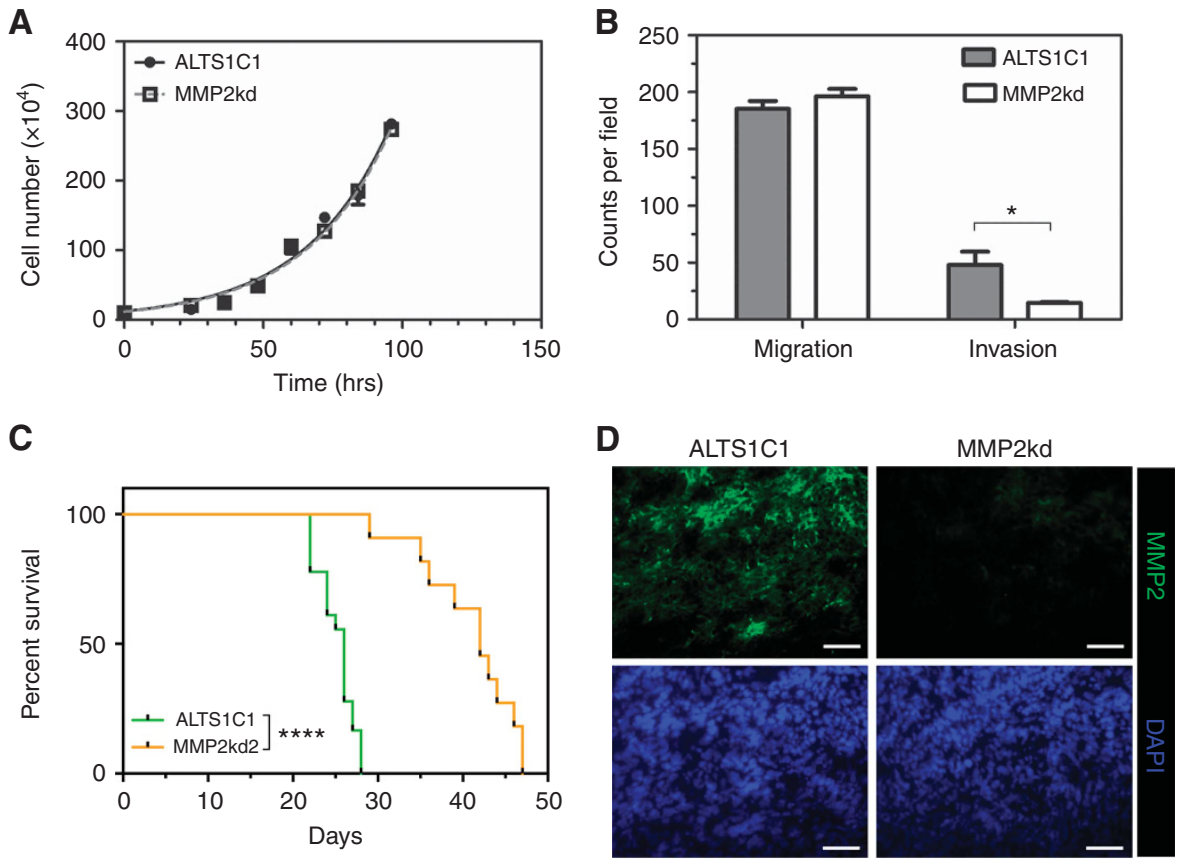

Figure 2. Effects of MMP2 inhibition on cell proliferation, cell invasion, and the survival of brain tumour-bearing mice. (A) In vitro cell proliferation rate had no difference between ALTS1C1 and MMP2kd cells. (B) MMP2 knockdown altered the invasive ability of ALTS1C1 cells, but not the migration ability. (C) Kaplan-Meier survival curves of ALTS1C1 $(n=18)$ and MMP2kd $(n=11)$ tumour-bearing mice. (D) Representative fluorescent images of MMP2 and DAPI staining in ALTS1C1 and MMP2kd tumours. Scale bar: $50 \mu \mathrm{m} .{ }^{\star} P<0.05,{ }^{\star * \star *} P<0.0001$.

A
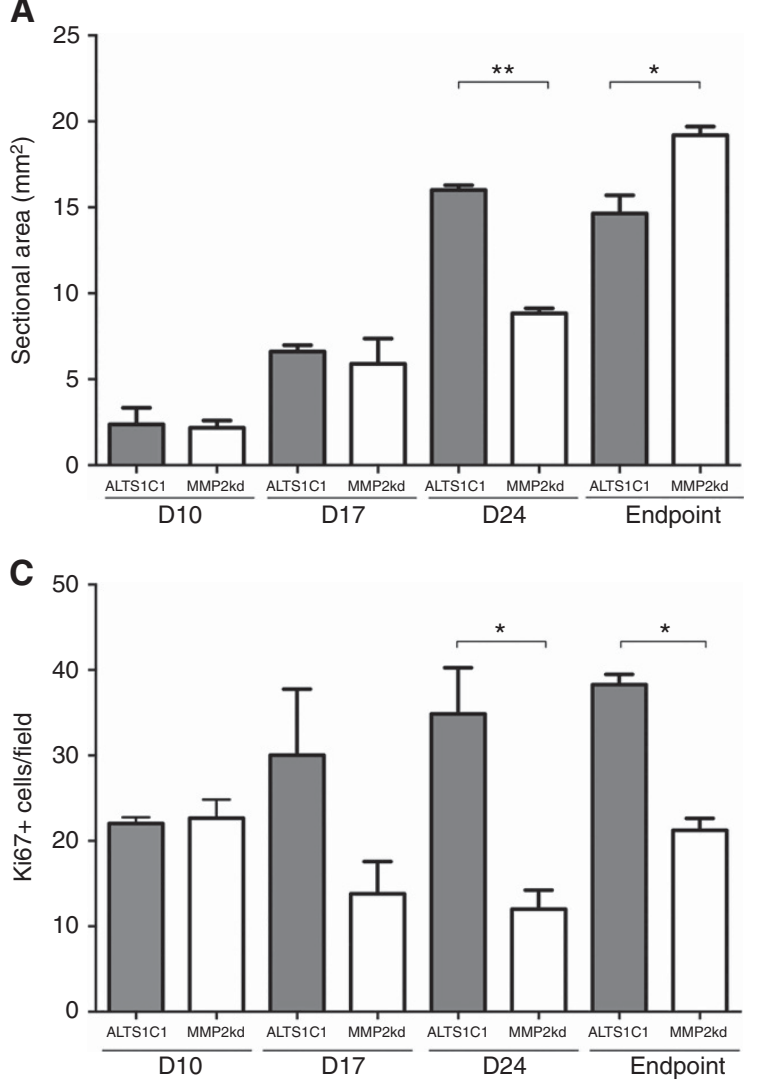

B

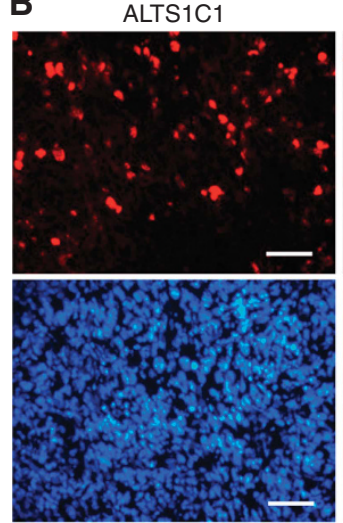

MMP2kd

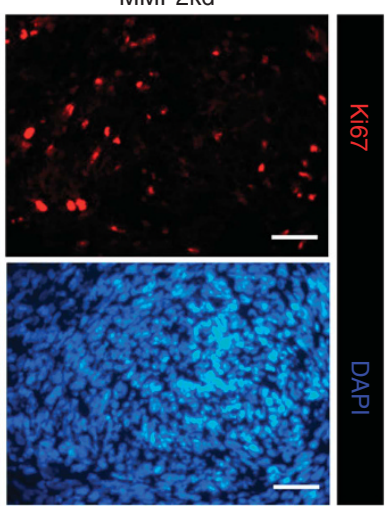

Figure 3. MMP2 knockdown reduced the proliferation rate in vivo. (A) The change of tumour size was followed at the indicated day after tumour inoculation. The mean sectional areas of tumours were measured at the maximum cross section with H\&E stain. (B) Representative images of Ki67 and DAPI staining in ALTS1C1 and MMP2kd tumours. Scale bar: $50 \mu \mathrm{m}$. (C) Quantification of Ki67 ${ }^{+}$cells was performed by counting the average of $\times 400$ magnification field for sections in each tissue. ${ }^{*} P<0.05,{ }^{* \star} P<0.01$. 
tumours might be eliminated with different pathological lesions from mice-bearing parental ALTS1C1 tumours. In other words, mice-bearing MMP2kd tumours died mainly from the pressure of an enlarged primary tumour and, in contrast, mice-bearing ALTS1C1 tumours likely died from the cause of a tumour infiltrating into critical brain regions.

Interestingly, when the expression of cell proliferation-associated antigen Ki67 in ALTS1C1 and MMP2kd tumours was compared (Figure 3B), the overall average number of $\mathrm{Ki}^{+} 7^{+}$cells in MMP2kd tumours (13.8 \pm 3.8 per field) on day 17 appeared to be less than that in ALTS1C1 tumours (30.0 \pm 7.7 per field; Figure 3C). However, MMP2kd tumours did present with a similar tumour size as ALTS1C1 tumours on day 17. The difference in the percentage of $\mathrm{Ki}^{+} 7^{+}$cells became more evident when the index was compared with tumours from day 24 mice (12.0 \pm 2.2 per field in MMP2kd tumour vs $38.3 \pm 1.2$ per field in ALTS1C1 tumours). Since most ALTS1C1 tumour-bearing mice were sick on day 24, the Ki67-proliferating index of MMP2kd tumours from endpoint mice was also measured. A slight increase $(21.2 \pm 1.4$ per field) was observed, but it was still lower than the number measured from that in ALTS1C1 tumours from endpoint mice. These results suggested that the knockdown of MMP2 expression decreased tumour cell proliferation in the brain despite its in vitro cell cycle time (Figure 2A) and tumour death marker (Supplementary Figure S4) was not changed.

MMP2 knockdown reduces the in vivo invasive ability of astrocytoma tumours. The above results showed that the suppression of MMP2 production could decrease the in vitro invasive (Figure $2 \mathrm{~B}$ ) and in vivo proliferating abilities of cancer cells (Figure $3 \mathrm{C}$ ). This suggested that the prolonged survival of mice-bearing MMP2kd tumours might be associated with the reduction of tumour invasiveness and tumour proliferation. To explore whether the in vivo invasiveness of ALTS1C1 tumours was diminished by MMP2 knockdown, the invasive ability of the tumour was verified by directly counting the number of infiltrating islands and measuring the intensity of GLUT-1 at the invading fronts. ALTS1C1 tumours displayed an apparent invasive pattern of invading the surrounding normal tissues during brain tumour growth; in contrast, MMP2kd tumours displayed a smoother border that made it easier to define the boundary between cancerous and normal tissues (Figure 4A). To quantify the invasiveness, the whole brain of each group was cut into serial sections at $100-\mu \mathrm{m}$ intervals and stained with DAPI. The infiltrating islands per section were then counted under microscopy. An island was scored as positive when the average diameter ((island length + island width)/2) was $\leqslant 200 \mu \mathrm{m}$ and when the island did not connect with the main tumour edge as described previously (Wang et al, 2012). Tumours from MMP2kd-bearing mice showed fewer infiltrating islands per section than tumours from ALTS1C1-bearing mice during the early (day 10: $11.6 \pm 2.3 \mathrm{vs}$ $21.6 \pm 3.1$ ), middle (day 17: $7.6 \pm 2.3$ vs $19.6 \pm 3.1$ ), the late stages (day 24: $10.5 \pm 2.3$ vs 17.6 \pm 3.7 ), and even in endpoint mice (endpoint: $8.6 \pm 2.0$ vs $19.0 \pm 2.2$; Figure $4 \mathrm{~B}$ ).

Not only the number of infiltrating islands decreased, but also the levels of the invasion marker GLUT-1 and the proliferation antigen Ki67 in the invading fronts were significantly reduced in MMP2kd tumours (Figure 4C and D). The smooth tumour margin, fewer infiltrating islands, and down-regulation of GLUT-1 expression in MMP2kd tumours revealed that the invasive ability of ALTS1C1 brain tumours was diminished when MMP2 gene expression was suppressed. This may be the key reason why micebearing MMP2kd tumours lived longer than mice-bearing parental ALTS1C1 tumours.

Inhibition of MMP2 expression impairs vascular function. Although it was clear that the suppression of MMP2 expression retarded tumour growth by reducing its invasive ability and cell proliferation rate, the in vitro results (Figure 2A) indicated that MMP2 levels within cancer cells had no direct effect on cell proliferation. We suspected that other tumour microenvironment factors might affect the in vivo tumour growth rate, as evidenced by the decrease in the Ki67 index (Figure 3C), but there was no change on death-associated index (Supplementary Figure S4).

In addition to being a direct contributor to matrix degradation in tumour invasion, MMP2 has also been implicated in tumour angiogenesis (Itoh et al, 1998; Vu et al, 1998; Fang et al, 2000; Zhou et al, 2000). To evaluate whether MMP2 played a role in tumour angiogenesis, the mean vascular density (MVD) of each tumour was measured by CD31 staining (Figure 5A). Tumour growth kinetics result (Figure 3A) revealed that MMP2kd tumour growth began to slow down after day 17; however, surprisingly, the MVD of MMP2kd tumours (day 17: $2.8 \pm 0.3$; day 24: $3.5 \pm 0.5$; and endpoint: $3.5 \pm 0.1$ ) showed no statistically significant difference when compared with parental ALTS1C1 tumours (day 17: $3.1 \pm 0.2$; day 24: $4.0 \pm 0.2$; and endpoint: $3.9 \pm 0.7$ ) throughout the tumour growth period up until the endpoint (Figure 5B). This finding implied that MMP2kd tumours might resort to other mechanisms to overcome the suppression of angiogenesis when MMP2 is deficient.

VEGF is a regulator for stimulating new vessel formation. The level of VEGF in tissues was examined by IHC staining and western blotting (Figure 5C and D), respectively. Interestingly, the level of VEGF expression $\left(0.34 \pm 0.01 \mathrm{ng} \mathrm{mg}^{-1}\right)$ quantified by ELISA (Supplementary Figure S5) in MMP2kd tumours was significantly higher than those in the normal brain $\left(0.24 \pm 0.01 \mathrm{ng} \mathrm{mg}^{-1}\right)$ and ALTS1C1 tumours $\left(0.31 \pm 0.01 \mathrm{ng} \mathrm{mg}^{-1}\right)$. This suggests that MMP2kd tumours had more VEGF proteins to overcome the suppression of angiogenesis when MMP2 activity was diminished. On the other hand, the cellular level of VEGF of MMP2kd cells was less than that in ALTS1C1 (Supplementary Figure S6). IHC double staining reveals that VEGF expression could be found in $\mathrm{F} 4 / 80^{+}$or $\mathrm{CD} 31^{+}$cells, but not in $\mathrm{Gr}-1^{+}, \mathrm{CD}^{+}$, and $\mathrm{CD}^{+}$cells (Supplementary Figure S7). Given the role of proteolytic release or cleavage on VEGF bioavailability by various proteolytic enzymes from various cellular sources (Hawinkels et al, 2008; Ito et al, 2009), the increase of VEGF proteins that was found in a complex MMP2kd tumour microenvironment, but not in in vitro MMP2kd cell cultures, is explainable.

The structure of vessels was further examined by staining the pericyte marker NG2 in ALTS1C1 and MMP2kd tumours (Figure 6), and the coverage of $\mathrm{NG} 2^{+}$pericytes on $\mathrm{CD} 31^{+}$ endothelial cells was used as an index for measuring the integrity of tumour vessels. The results showed that ALTS1C1 tumours not only had a higher level of NG2 expression than the MMP2kd tumours (Figure 6A), but also a higher percentage of $\mathrm{CD} 31^{+}$ $\mathrm{NG}^{+}$cells at either day $17(31.6 \pm 0.9)$, day $24(29.9 \pm 0.7)$, or at an endpoint $(29.1 \pm 0.3)$ than those in MMP2kd tumours $(11.3 \pm 1.2,14.6 \pm 2.4$, and $16.6 \pm 1.5$ at day17, day 24 , and at an endpoint, respectively; Figure 6B). These results suggested that vessels in MMP2kd tumours were generally less mature than those in ALTS1C1 tumours, indicating that the vascular function in MMP2kd tumours might similarly be less. Vascular function in both tumours was further examined by Hoechst33342 dye perfusion. Although the perfusion of vessels was heterogeneous in both tumours (upper panel of Figure 6C), it did show that the overall perfusion areas in ALTS1C1 tumours were larger than those in MMP2kd tumours (Figure 6D). The lower perfusion of Hoechst33342 dye in MMP2kd tumours correlated with a higher percentage of the PIMO-staining area in these tumours (lower panel of Figure $6 \mathrm{C}$ and D). In summary, these results indicated that MMP2 suppression could hinder the recruitment of $\mathrm{NG} 2^{+}$ pericytes and result in poor blood perfusion and could consequently retard tumour growth. 
A
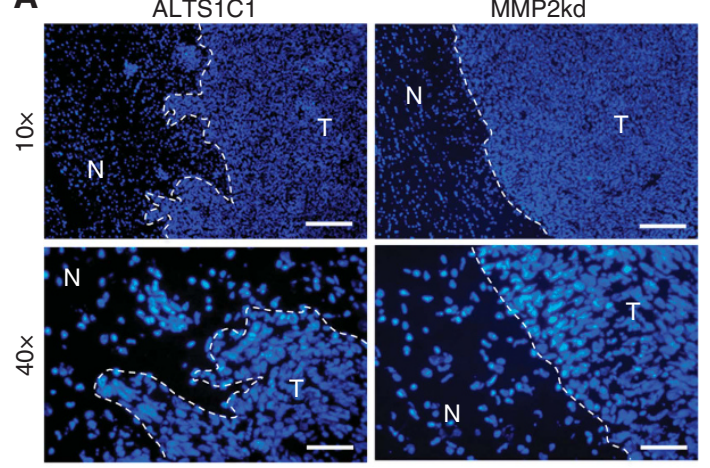

C
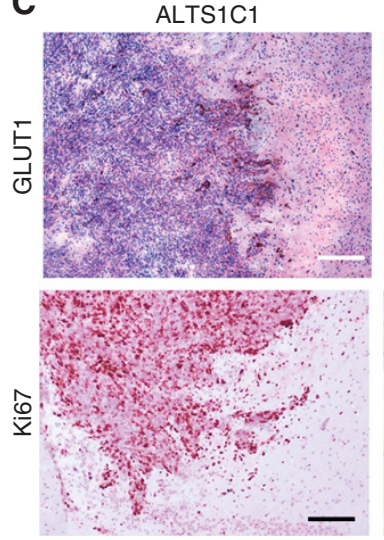

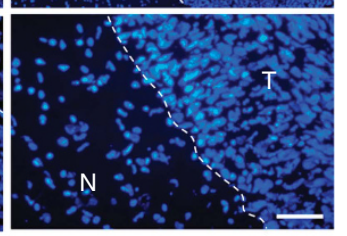

MMP2kd

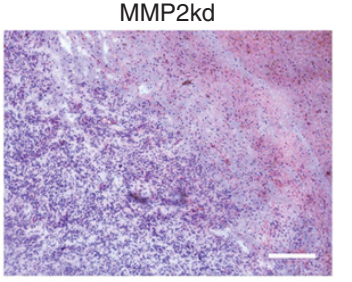

B

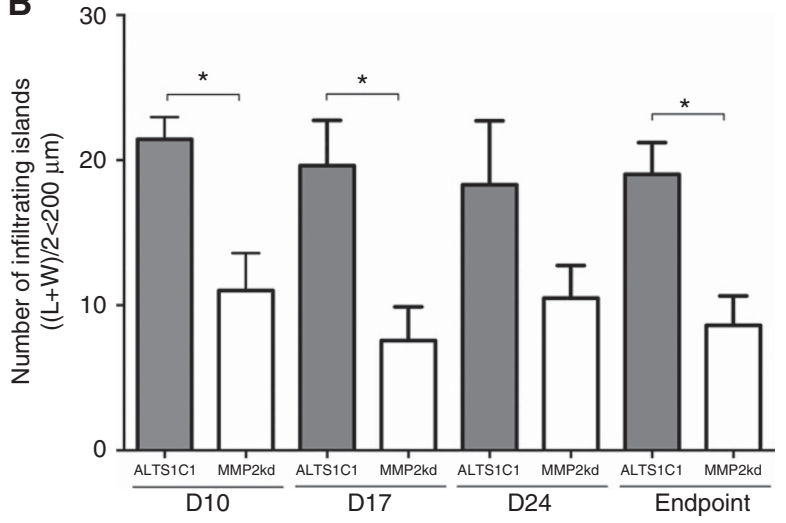

D

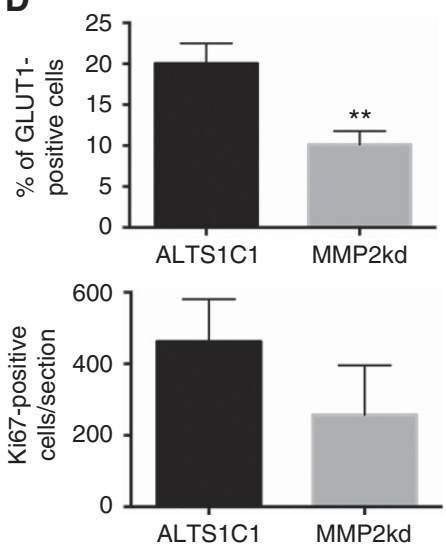

Figure 4. MMP2 expression promoted tumour invasion. (A) Representative images of tumour borders stained with DAPI in ALTS1C1 and MMP2kd tumours from day 17 tissues. ALTS1C1 had an invasive front and small tumour-infiltrating islands, but MMP2kd tumours had smooth boundaries and few islands. $T$ indicated the local tumour site and $\mathrm{N}$ indicated the normal brain tissue. (B) Number of infiltrating islands in the brain was counted at the indicated day. The plot represents the average island numbers of three brains on each group observed under $\times 100$ magnification and repeated in three independent experiments. (C) Representative images of GLUT-1 (upper panel) and Ki67 (lower panel) expression in the tumour margin of ALTS1C1 and MMP2kd tumours from day 17 tissues. The nucleus was visualised with haematoxylin staining (blue). (D) The quantification data for the comparison of GLUT-1 and Ki67 in the tumour margin of ALTS1C1 and MMP2kd tumours. ${ }^{\star} P<0.05,{ }^{\star \star} P<0.01$.

\section{DISCUSSION}

The invasion of brain tumour cells into the surrounding normal brain regions is the major cause of unsuccessful treatments for HGG. We have previously reported that a murine anaplastic astrocytoma cell-line, ALTS1C1, recaptures the invading features of malignant brain tumours. The invading features are associated with an up-regulated SDF-1 expression through its effect on tumour-associated macrophages (Wang et al, 2012). In this study, we further report that the invading features in ALTS1C1 tumours are also associated with tumour cell-derived MMP2 production because of its effect on cell invasion and the vessel formation mechanism.

The association between higher MMP2 expression levels with brain tumour malignancies has been reported in many clinical cases (Wang et al, 2003; Komatsu et al, 2004). In this study, we took advantage of using the murine orthotopic astrocytoma tumour model to illustrate that MMP2 expression at the leading invasion fronts was associated with the invasive marker GLUT-1 expression. Furthermore, the invasion-associated features were diminished when cancer cell-derived MMP2 was knocked down by shRNA. Our findings indicate that cancer cell-derived MMP2 plays a leading role in tumour invasion into the adjacent normal brain tissue and this is further supported by an in vitro assay showing that the invasive ability of tumour cells was decreased in MMP2kd cells. Since the mobility of cancer cells is not altered in MMP2kd cells, it seems that the major role of MMP2 on tumour invasion is associated with its ability to degrade the ECM (McCawley and Matrisian, 2001).

The tumour growth kinetic data show that the knockdown of MMP2 expression has no effect on initial tumour growth until 17 days after tumour inoculation. This indicates that the initial tumour growth mainly depends on the intrinsic tumour cell cycle time, which could be MMP2 independent since the cell cycle time is not altered in MMP2kd cells. When tumours grow to a critical volume and need to expand outwards, the involvement of MMP2 on ECM degradation becomes essential for tumour expansion and invasion. This explains why the trend of tumour growth and cellular proliferation in MMP2kd tumours did not decrease until 17 days after tumour inoculation. This may also explain why the expression of MMP2 at tumour-invading fronts is higher than that in the tumour center. A recent publication showed that the different roles of bone marrow-derived progenitor cells (BMDCs) at different stages may be associated with a distinct molecular mechanism of neovascularisation at different regions of different states of the GBM model (Burrell et al, 2014). Our study showed that in addition to tumour cell-derived MMP2, MMP2 expression was also found to co-localise with $\mathrm{CD} 31^{+}$vessels at the invading tumour front and infiltrating islands, but not in the tumour central region (Figure 1). This not only illustrates that there are multiple MMP2-producing cells in tumours, but also that the unusual microenvironments of invading tumour fronts have evoked cascade signalling for MMP2 expression. 
A

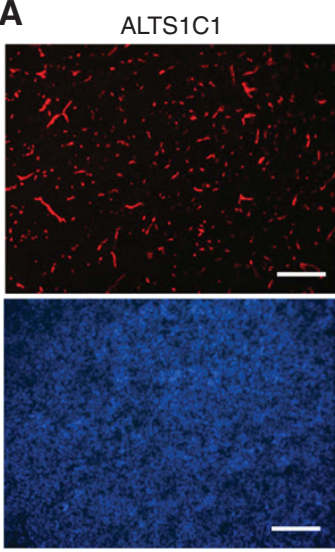

C
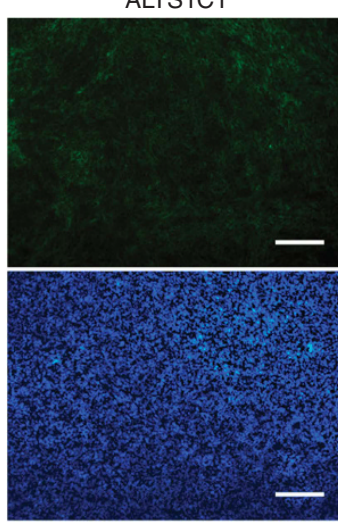

MMP2kd

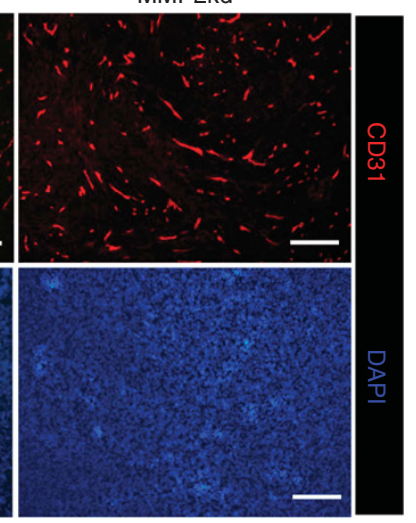

MMP2kd

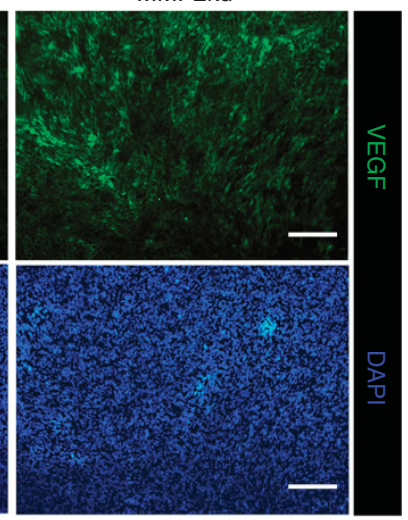

B
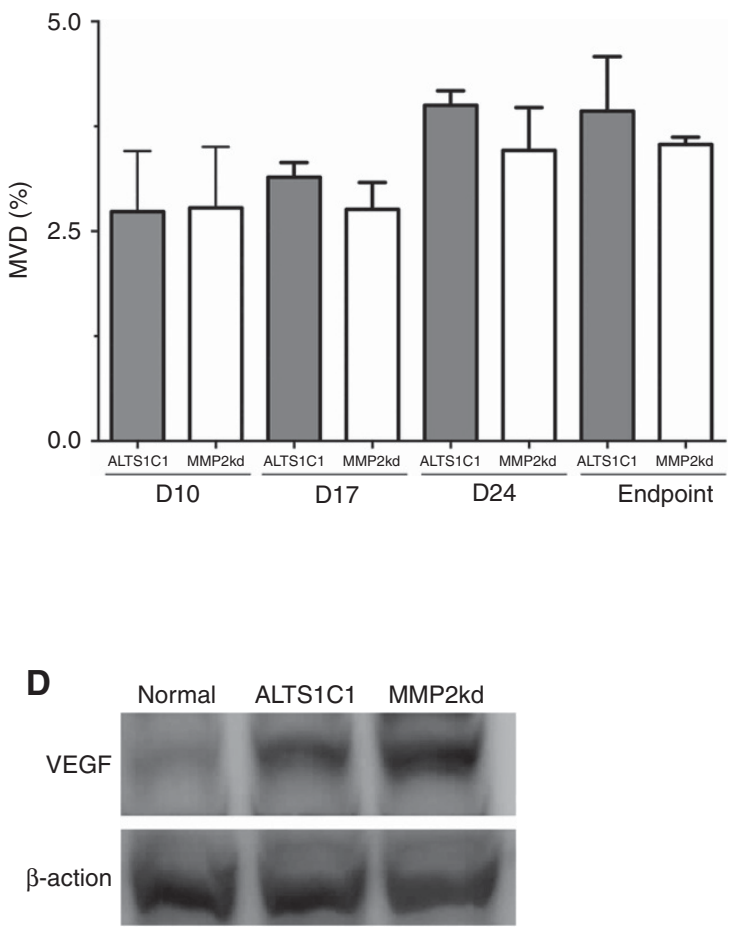

Figure 5. Suppression of MMP2 expression in tumour cells did not affect MVD, but increased VEGF expression. (A) Representative images of vessel density in tumours. Scale bar: $200 \mu \mathrm{m}$. (B) Quantification of MVD at the indicated day after tumour implantation. (C) Higher level of VEGF in MMP2kd tumours was detected by IHC staining. Scale bar: $200 \mu \mathrm{m}$. (D) The level of VEGF in normal brain, ALTS1C1, and MMP2kd tumour lysates was measured by western blotting.

In addition to their role in degrading the ECM, this study describes that MMP2 also plays a role in vessel formation. We have previously shown that the MVD is higher in the invading fronts of ALTS1C1 tumours (Wang et al, 2012). A recent study using two-photon laser microscopy coupled with an intracranial window in mouse models of GBM and bone marrow transplantation clearly showed that GBMs rely on diverse molecular mechanisms for neovascularisation in the peripheral versus central regions when tumours grew beyond a critical volume (Burrell et al, 2014). In this study, we demonstrate that some high-intensity MMP2-expressing cells are co-localised with $\mathrm{CD} 1^{+}$vessels at the invading fronts and infiltrating islands but not in the tumour centre. These invasion-associated vessels also express a high intensity of GLUT-1 antigen. The increase in GLUT-1 expression at the tumour invasion site has been reported in many tumours (Younes et al, 1995; Furudoi et al, 2001). The enhancement of GLUT-1 expression signified an increased glucose uptake (high glycolysis) that is correlated with acid production. Recent reports have shown that acidic $\mathrm{pH}$ environments can promote the local invasive tumour growth and metastasis (Estrella et al, 2013). This study shows that GLUT-1 expression was increased not only in tumour cells but also in the vessels within the invading tumour fronts. These GLUT-1+ vessels also expressed MMP2, which was independent of tumour cell-derived MMP2 (Supplementary Figure S8) and was instead associated with the unique tumour microenvironment of invading tumour fronts. Whether these MMP2 ${ }^{+}$or GLUT- ${ }^{+}$ vessels are formed through the assistance of a new perivascular BMDC (Burrell et al, 2014) is an interesting question requiring further study.
It appears that an unusual microenvironment has been developed in the invading tumour fronts of malignant glioma. Our previous study on this tumour model has shown that the number of F4/80 ${ }^{+}$TAMs is significantly increased at the tumour fronts and infiltrating islands (Wang et al, 2012). A recent study demonstrated that more BMDCs were recruited to the peripheral regions than to the central regions (Burrell et al, 2014). In the context of these findings, this study describes that the expression of MMP2 and GLUT-1 is specifically activated at the invading tumour fronts. It has been reported that factors released from TAMs could induce MMP2 expression in several types of cancers (Bredin et al, 2003; Kuwada et al, 2003). When tissues with SDF knockdown tumours were stained for MMP2 expression, we found that MMP2 expression in the invading tumour front was also reduced (Supplementary Figure S9A) in contrast to the number of TAMs that was not significantly altered in MMP2kd tumours (Supplementary Figure S9B). Taken together, these two studies illustrate how anaplastic astrocytoma cancer cells promote tumour invasiveness. This path relies on cancer cells that secrete SDF-1 to recruit BMDCs. The recruited BMDCs then become activated TAMs and interact with tumour cells and endothelial cells to enhance the expression and activity of MMP2 and GLUT-1 to promote tumour invasiveness.

Reports show that MMP2 levels might be associated with the process of tumour angiogenesis (Gatto et al, 1999; Martin et al, 1999; Oh et al, 2001; Rodriguez-Manzaneque et al, 2001). However, we found that the overall vessel density in MMP2kd tumours was not changed. In contrast, in vivo proliferation assays showed that the Ki67 index in MMP2kd tumours began to decrease 17 days after tumour inoculation, which was consistent 
A

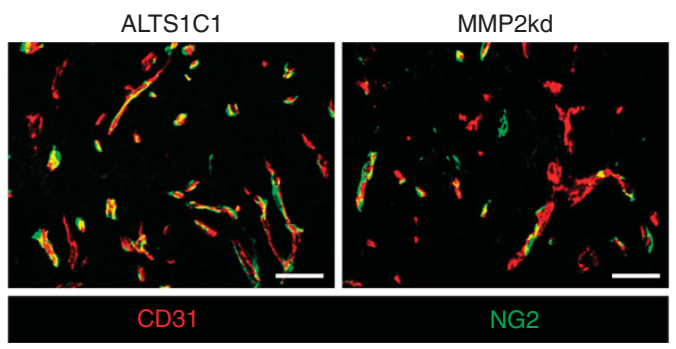

C
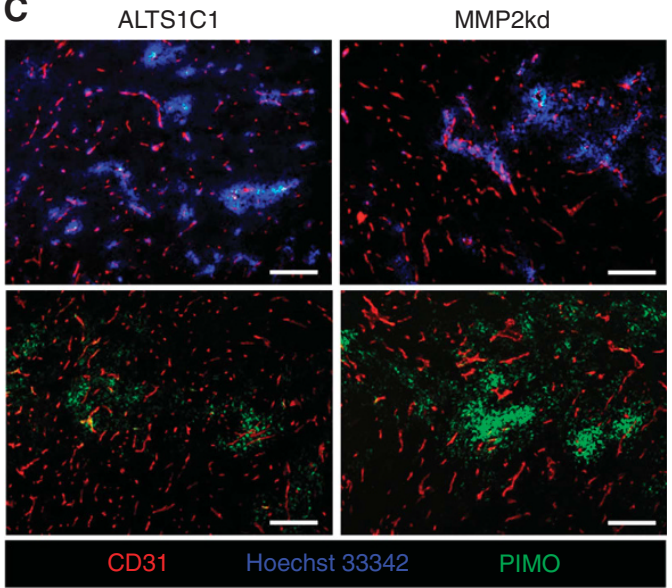

Figure 6. MMP2 expression was associated with vascular function. (A) Representative images of ALTS1C1 and MMP2kd tumours stained with CD31 and NG2. Scale bar: $50 \mu \mathrm{m}$. (B) Quantification of the ratio of NG2 covered on the blood vessels at the indicated day after tumour implantation. (C) Representative images of blood perfusion and hypoxia in ALTS1C1 and MMP2kd tumours. CD31 and PIMO were stained to identify vessels and tumour hypoxia, respectively, and the blood perfusion was visualised using Hoechst33342. Scale bar: $200 \mu \mathrm{m}$.

(D) Quantification of the blood perfusion (left panel) and hypoxia area (right panel) in tumours. ${ }^{\star} P<0.05,{ }^{\star} * 2<0.01$.

with the time that the growth rate of MMP2kd tumours began to fall behind parental tumours. The mismatch between tumour growth rate and MVD indicates that the vessel formation mechanism in MMP2kd tumours may not be the same as that in parental ALTS1C1 tumours. Indeed, we found an increased VEGF expression in MMP2kd tumours (in vivo), but not in MMP2kd cells (in vitro), as well as less-mature vessels with a lower perfusion efficiency that was associated with a higher hypoxia ratio than that in parental tumours. These indicate that some mechanisms in MMP2kd tumours were switched on to induce VEGF expression to promote angiogenesis when MMP2 activity decreased. The decrease of pericyte coverage on blood vessels in MMP2kd tumours suggests that MMP2, like MMP-9 (Du et al, 2008), also has a role in pericyte recruitment (Du et al, 2008). In the MMP-9 model, it has been suggested that the recruitment of bone marrow-derived-pericytes to glioma depended on MMP-9 expression of CD $45^{+}$vascular modulatory cells recruited to tumours by the SDF-1/CXCR4 axis. It is likely that a similar mechanism may be applied to the action of MMP2 upon pericyte recruitment because we found that ALTS1C1derived SDF-1 production was essential for BMDC recruitment and MMP2 expression.

In summary, this study illustrates a dual role for MMP2 in cancer progression, suggesting that MMP2 promotes tumour invasiveness by its ability to degrade ECM without affecting cell motility. In addition, MMP2 can also stimulate tumour growth by promoting vessel maturation and function. Therefore, MMP2, in addition to MMP-9, is also a potential target molecule for treating malignant brain tumours.

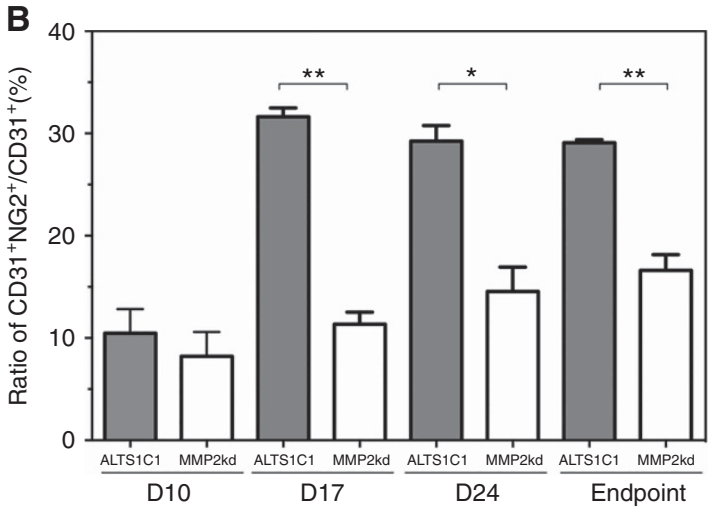

D

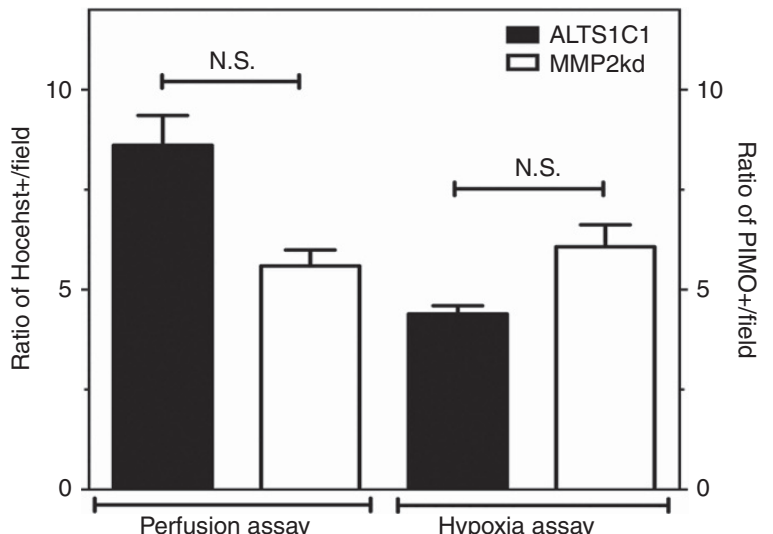

ACKNOWLEDGEMENTS

This work is supported by grants NSC102-2314-B-007-003-MY3, NHRI-EX106-10514BI, and NTHU-105N710CK6 to C-SC, CMRPG3E1301-2 and MOST104-2314-B-182A-087 to J-HH, and CIRPD1D0061-63 to F-H C.

\section{CONFLICT OF INTEREST}

The authors declare no conflict of interest.

\section{REFERENCES}

Blumenthal DT, Gorlia T, Gilbert MR, Kim MM, Burt Nabors L, Mason WP, Hegi ME, Zhang P, Golfinopoulos V, Perry JR, Hyun Nam D, Erridge SC, Corn BW, Mirimanoff RO, Brown PD, Baumert BG, Mehta MP, van den Bent MJ, Reardon DA, Weller M, Stupp R (2017) Is more better? The impact of extended adjuvant temozolomide in newly diagnosed glioblastoma: a secondary analysis of EORTC and NRG Oncology/RTOG. Neuro Oncol 19(8): 1119-1126.

Bredin CG, Liu Z, Klominek J (2003) Growth factor-enhanced expression and activity of matrix metalloproteases in human non-small cell lung cancer cell lines. Anticancer Res 23(6C): 4877-4884.

Burrell K, Singh S, Jalali S, Hill RP, Zadeh G (2014) VEGF regulates regionspecific localization of perivascular bone marrow-derived cells in glioblastoma. Cancer Res 74(14): 3727-3739.

Chiang CS, Fu SY, Wang SC, Yu CF, Chen FH, Lin CM, Hong JH (2012) Irradiation promotes an M2 macrophage phenotype in tumor hypoxia. Front Oncol 2(1): 89-100. 
Coussens LM, Werb Z (1996) Matrix metalloproteinases and the development of cancer. Chem Biol 3(11): 895-904.

Droppelmann CA, Gutierrez J, Vial C, Brandan E (2009) Matrix metalloproteinase-2-deficient fibroblasts exhibit an alteration in the fibrotic response to connective tissue growth factor/CCN2 because of an increase in the levels of endogenous fibronectin. J Biol Chem 284(20): 13551-13561.

Du R, Petritsch C, Lu K, Liu P, Haller A, Ganss R, Song H, Vandenberg S, Bergers G (2008) Matrix metalloproteinase-2 regulates vascular patterning and growth affecting tumor cell survival and invasion in GBM. Neuro Oncol 10(3): 254-264.

Egeblad M, Werb Z (2002) New functions for the matrix metalloproteinases in cancer progression. Nat Rev Cancer 2(3): 161-174.

Estrella V, Chen T, Lloyd M, Wojtkowiak J, Cornnell HH, Ibrahim-Hashim A, Bailey K, Balagurunathan Y, Rothberg JM, Sloane BF, Johnson J, Gatenby RA, Gillies RJ (2013) Acidity generated by the tumor microenvironment drives local invasion. Cancer Res 73(5): 1524-1535.

Fang J, Shing Y, Wiederschain D, Yan L, Butterfield C, Jackson G, Harper J, Tamvakopoulos G, Moses MA (2000) Matrix metalloproteinase-2 is required for the switch to the angiogenic phenotype in a tumor model. Proc Natl Acad Sci USA 97(8): 3884-3889.

Forsyth PA, Wong H, Laing TD, Rewcastle NB, Morris DG, Muzik H, Leco KJ, Johnston RN, Brasher PM, Sutherland G, Edwards DR (1999) Gelatinase-A (MMP-2), gelatinase-B (MMP-9) and membrane type matrix metalloproteinase-1 (MT1-MMP) are involved in different aspects of the pathophysiology of malignant gliomas. Br J Cancer 79(11-12): 1828-1835.

Frankowski H, Gu YH, Heo JH, Milner R, Del Zoppo GJ (2012) Use of gel zymography to examine matrix metalloproteinase (gelatinase) expression in brain tissue or in primary glial cultures. Methods Mol Biol 814: 221-233.

Furudoi A, Tanaka S, Haruma K, Yoshihara M, Sumii K, Kajiyama G, Shimamoto F (2001) Clinical significance of human erythrocyte glucose transporter 1 expression at the deepest invasive site of advanced colorectal carcinoma. Oncology 60(2): 162-169.

Gatto C, Rieppi M, Borsotti P, Innocenti S, Ceruti R, Drudis T, Scanziani E, Casazza AM, Taraboletti G, Giavazzi R (1999) BAY 12-9566, a novel inhibitor of matrix metalloproteinases with antiangiogenic activity. Clin Cancer Res 5(11): 3603-3607.

Gerg M, Kopitz C, Schaten S, Tschukes A, Kahlert C, Stangl M, von Weyhern CW, Brucher BL, Edwards DR, Brand K, Kruger A (2008) Distinct functionality of tumor cell-derived gelatinases during formation of liver metastases. Mol Cancer Res 6(3): 341-351.

Giese A, Bjerkvig R, Berens ME, Westphal M (2003) Cost of migration: invasion of malignant gliomas and implications for treatment. J Clin Oncol 21(8): 1624-1636.

Guo P, Imanishi Y, Cackowski FC, Jarzynka MJ, Tao HQ, Nishikawa R, Hirose T, $\mathrm{Hu}$ B, Cheng SY (2005) Up-regulation of angiopoietin-2, matrix metalloprotease-2, membrane type 1 metalloprotease, and laminin 5 gamma 2 correlates with the invasiveness of human glioma. Am J Pathol 166(3): 877-890,

Hawinkels LJ, Zuidwijk K, Verspaget HW, de Jonge-Muller ES, van Duijn W, Ferreira V, Fontijn RD, David G, Hommes DW, Lamers CB, Sier CF (2008) VEGF release by MMP-9 mediated heparan sulphate cleavage induces colorectal cancer angiogenesis. Eur J Cancer 44(13): 1904-1913.

Ito T-KK, Ishii G, Saito S, Yano K, Hoshino A, Suzuki T, Ochiai A (2009) Degradation of soluble VEGF receptor-1 by MMP-7 allows VEGF access to endothelial cells. Blood 113(10): 2363-2369.

Itoh T, Tanioka M, Yoshida H, Yoshioka T, Nishimoto H, Itohara S (1998) Reduced angiogenesis and tumor progression in gelatinase A-deficient mice. Cancer Res 58(5): 1048-1051.

Komatsu K, Nakanishi Y, Nemoto N, Hori T, Sawada T, Kobayashi M (2004) Expression and quantitative analysis of matrix metalloproteinase-2 and -9 in human gliomas. Brain Tumor Pathol 21(3): 105-112.

Kuwada Y, Sasaki T, Morinaka K, Kitadai Y, Mukaida N, Chayama K (2003) Potential involvement of IL- 8 and its receptors in the invasiveness of pancreatic cancer cells. Int J Oncol 22(4): 765-771.

Lampert K, Machein U, Machein MR, Conca W, Peter HH, Volk B (1998) Expression of matrix metalloproteinases and their tissue inhibitors in human brain tumors. Am J Pathol 153(2): 429-437.

Martin DC, Sanchez-Sweatman OH, Ho AT, Inderdeo DS, Tsao MS, Khokha R (1999) Transgenic TIMP-1 inhibits simian virus 40T antigeninduced hepatocarcinogenesis by impairment of hepatocellular proliferation and tumor angiogenesis. Lab Invest 79(2): 225-234.
McCawley LJ, Matrisian LM (2001) Matrix metalloproteinases: they're not just for matrix anymore! Curr Opin Cell Biol 13(5): 534-540.

Mosig RA, Dowling O, DiFeo A, Ramirez MC, Parker IC, Abe E, Diouri J, Aqeel AA, Wylie JD, Oblander SA, Madri J, Bianco P, Apte SS, Zaidi M, Doty SB, Majeska RJ, Schaffler MB, Martignetti JA (2007) Loss of MMP-2 disrupts skeletal and craniofacial development and results in decreased bone mineralization, joint erosion and defects in osteoblast and osteoclast growth. Hum Mol Genet 16(9): 1113-1123.

Oh J, Takahashi R, Kondo S, Mizoguchi A, Adachi E, Sasahara RM, Nishimura S, Imamura $\mathrm{Y}$, Kitayama H, Alexander DB, Ide C, Horan TP, Arakawa T, Yoshida H, Nishikawa S, Itoh Y, Seiki M, Itohara S, Takahashi C, Noda M (2001) The membrane-anchored MMP inhibitor RECK is a key regulator of extracellular matrix integrity and angiogenesis. Cell 107(6): 789-800.

Omuro A, DeAngelis LM (2013) Glioblastoma and other malignant gliomas: a clinical review. JAMA 310(17): 1842-1850.

Rao JS (2003) Molecular mechanisms of glioma invasiveness: the role of proteases. Nat Rev Cancer 3(7): 489-501.

Ray JM, Stetler-Stevenson WG (1994) The role of matrix metalloproteases and their inhibitors in tumour invasion, metastasis and angiogenesis. Eur Respir J 7(11): 2062-2072.

Rodriguez-Manzaneque JC, Lane TF, Ortega MA, Hynes RO, Lawler J, IruelaArispe ML (2001) Thrombospondin-1 suppresses spontaneous tumor growth and inhibits activation of matrix metalloproteinase-9 and mobilization of vascular endothelial growth factor. Proc Natl Acad Sci USA 98(22): 12485-12490.

Rosenberg GA (1995) Matrix metalloproteinases in brain injury. J Neurotrauma 12(5): 833-842.

Sawaya RE, Yamamoto M, Gokaslan ZL, Wang SW, Mohanam S, Fuller GN, McCutcheon IE, Stetler-Stevenson WG, Nicolson GL, Rao JS (1996) Expression and localization of $72 \mathrm{kDa}$ type IV collagenase (MMP-2) in human malignant gliomas in vivo. Clin Exp Metastasis 14(1): 35-42.

Stetler-Stevenson WG (1990) Type IV collagenases in tumor invasion and metastasis. Cancer Metastasis Rev 9(4): 289-303.

Stupp R, Taillibert S, Kanner AA, Kesari S, Steinberg DM, Toms SA, Taylor LP, Lieberman F, Silvani A, Fink KL, Barnett GH, Zhu JJ, Henson JW, Engelhard HH, Chen TC, Tran DD, Sroubek J, Tran ND, Hottinger AF, Landolfi J, Desai R, Caroli M, Kew Y, Honnorat J, Idbaih A, Kirson ED, Weinberg U, Palti Y, Hegi ME, Ram Z (2015) Maintenance therapy with tumor-treating fields plus temozolomide vs temozolomide alone for glioblastoma: a randomized clinical trial. JAMA 314(23): 2535-2543.

Vehlow A, Cordes N (2013) Invasion as target for therapy of glioblastoma multiforme. Biochim Biophys Acta 1836(2): 236-244.

Vu TH, Shipley JM, Bergers G, Berger JE, Helms JA, Hanahan D, Shapiro SD, Senior RM, Werb Z (1998) MMP-9/gelatinase B is a key regulator of growth plate angiogenesis and apoptosis of hypertrophic chondrocytes. Cell 93(3): 411-422.

Wang M, Wang T, Liu S, Yoshida D, Teramoto A (2003) The expression of matrix metalloproteinase-2 and -9 in human gliomas of different pathological grades. Brain Tumor Pathol 20(2): 65-72.

Wang SC, Hong JH, Hsueh C, Chiang CS (2012) Tumor-secreted SDF-1 promotes glioma invasiveness and TAM tropism toward hypoxia in a murine astrocytoma model. Lab Invest 92(1): 151-162.

Wang SC, Yu CF, Hong JH, Tsai CS, Chiang CS (2013) Radiation therapyinduced tumor invasiveness is associated with SDF-1-regulated macrophage mobilization and vasculogenesis. PLoS One 8(8): e69182.

Younes M, Brown RW, Mody DR, Fernandez L, Laucirica R (1995) GLUT1 expression in human breast carcinoma: correlation with known prognostic markers. Anticancer Res 15(6B): 2895-2898.

Zhou Z, Apte SS, Soininen R, Cao R, Baaklini GY, Rauser RW, Wang J, Cao Y, Tryggvason K (2000) Impaired endochondral ossification and angiogenesis in mice deficient in membrane-type matrix metalloproteinase I. Proc Natl Acad Sci USA 97(8): 4052-4057.

(c) (1)(2) This work is licensed under the Creative Commons (c) ${ }_{\text {BY }}$ NC $\mathrm{SA}$ Attribution-Non-Commercial-Share Alike 4.0 International License. To view a copy of this license, visit http:// creativecommons.org/licenses/by-nc-sa/4.0/

(C) The Author(s) named above 2017

Supplementary Information accompanies this paper on British Journal of Cancer website (http://www.nature.com/bjc) 